\title{
Sensing cell mechanics with atomic force microscopy
}

\section{Editorial}

Cell, as the basic unit of human life, has a biologically complex system. The mechanical property of biological cells is one of the most prominent properties, which is closely related to their physiological performance. Living cells possess specific physical and structural properties, enabling them to maintain functional in physiological environment. Cell mechanics plays a major role in many cell physiological events such as cell differentiation, cell migration and cell deformability. ${ }^{1}$ Deviations from normal values of biomechanical properties of cells will undermine not only the physical integrity of the cells, but also their biological functions. Abnormal tissue stiffness is often an indication of a wide range of diseases such as osteoporosis, ${ }^{2}$ atherosclerosis, ${ }^{3}$ caner, ${ }^{4}$ etc. Clinically, the mechanics of tissue or organ has been used for diagnosis purpose. Palpation is used to diagnose the abnormal hardness of tissue caused by diseases like breast cancer.

Elastographic techniques, such as ultrasound elastography ${ }^{5}$ and Magnetic resonance elastography, ${ }^{6}$ have attracted much clinical interest in the ability of diagnosing diseases based on analyzing tissue mechanical properties. Although these methods can somehow provide quantitative stiffness information, the accuracy is not sufficient especially when the disease is at an early stage or a small scale. Cell mechanics has become an important biomarker for diseases. Studies are attempting to find a correlation of cellular elasticity with cell functions and human diseases, since changes in pathophysiological properties of tissues are often manifested at the cellular level. Understanding of cell mechanics is critical to provide potential clinical methods that will enhance detection, diagnosis, and treatment of diseases.

There are a number of advanced techniques to measure cellular mechanics, including Atomic Force Microscopy (AFM), infrared laser traps (optical tweezers), magnetic tweezers, micropipette aspiration, magnetic twisting cytometry, optical stretching. Among them, AFM is one of the most versatile techniques in mechanobiology. Thanks to its high accuracy, AFM allows to obtain a high resolution image at nanoscale. In particular, AFM possesses several advantages over other measuring techniques. Regardless of high resolution, AFM can operate in both ambient conditions and aqueous environment, which could study biological materials in biophysical condition nondestructively. ${ }^{7}$ It has overcome the limitation of scanning tunneling microscope to carry out measurement at physiological environment, which has shown immense impact on force-based probing of biological materials. ${ }^{8}$ Currently, AFM has been used in a wide range of applications, such as imaging, characterization of surface property, micromanipulation, force study and molecular interaction study.

In addition to the extraordinary capability of imaging cellular microenvironments, surface structure, and sub-cellular structure, AFM acts as a state-of-the-art tool to probe biomechanical properties of biological materials to extract quantitative parameters of both biological systems and non-biological systems. ${ }^{9}$ AFM-based force spectroscopy can detect the changes in cell elasticity to distinguish
Volume 2 Issue 2 - 2017

\author{
Qiuquan Guo \\ Department of Mechanical \& Materials Engineering, Biomedical \\ Engineering, Western University, Canada
}

\begin{abstract}
Correspondence: Qiuquan Guo, Department of Mechanical \& Materials Engineering, Biomedical Engineering, Western University, II5I Richmond Street, London, Ontario, N6A 3K7, Canada; Email qguo29@uwo.ca
\end{abstract}

Received: March 19, 2017 | Published: March 22, 2017

metastatic cells from benign cells, thus identifying disease states in cancer. ${ }^{10-12}$ What is more, AFM is applicable to detection on the pathological changes of cartilage cells in osteoarthritis. ${ }^{13}$ All above demonstrate that AFM can be potentially developed as a diagnostic tool for detecting human disease states with fairly high efficiency and accuracy.

With the unprecedented signal-to-noise ratio, AFM enables both the imaging of biological interfaces from the cellular to the molecular scale and force measurement at nanoresolution. Combining the imaging mode and force mode, Guo has correlated the mechanical property with cell morphology. ${ }^{14}$ Furthermore, AFM has been integrated with other state-of-the-art technology to provide more informative properties in the cells. Together with fluorescent image, the real-time assembly and distribution of stress fibers, acting as cytoskeleton of cells, were investigated. ${ }^{12}$ The organization of actin fibers has significant effect on the Stiffness, which has been found to have correlation with healthy and cancer cells. ${ }^{15}$

There is no doubt that AFM-based technology has revolutionized nanotechnology in biological systems. With more research results from both AFM and other hybrid AFM studies, they have substantiated that cell mechanics can provide as a diagnosis tool for healthy and diseased cells. While efforts are still required for future clinical applications. Although AFM technology has matured into a powerful nanoscopic platform, the throughput is still required to be improved. In addition, the future miniaturization of AFM force-based technology is essential to be used as a clinical tool, even for in vivo use.

\section{Acknowledgements}

None.

\section{Conflict of interest}

The author declares no conflict of interest.

\section{References}

1. Lim CT, Zhou EH, Quek ST. Mechanical models for living cells-a review. J Biomech. 2006;39(2):195-216.

2. Langton CM, Njeh CF, Hodgskinson R, et al. Prediction of mechanical properties of the human calcaneus by broadband ultrasonic attenuation. Bone. 1996;18(6):495-503. 
3. Rourke M. Mechanical principles in arterial disease. Hypertension. 1995;26(1):2-9.

4. Faria EC, Ma N, Gazi E, et al. Measurement of elastic properties of prostate cancer cells using AFM. Analyst. 2008;133(11):1498-1500.

5. Piscaglia F, Marinelli S, Bota S, et al. The role of ultrasound elastographic techniques in chronic liver disease: current status and future perspectives. Eur J Radiol. 2014;83(3):450-455.

6. Manduca A, Oliphant TE, Dresner MA, et al. Magnetic resonance elastography: non-invasive mapping of tissue elasticity. Medical Image Analysis. 2001;5(4):237-254.

7. Hörber JK, Häberle W, Ohnesorge F, et al. Investigation of living cells in the nanometer regime with the scanning force microscope. Scanning Microsc. 1992;6(4):919-929.

8. Jena BP, Hörber JK. Atomic force microscopy in cell biology. 1st ed. USA: Academic Press; 2002. 415 p.

9. Alsteens D, Gaub HE, Newton R, et al. Atomic force microscopy-based characterization and design of biointerfaces. Nature Reviews Materials. 2017;2:17008.
10. Cross SE, Jin YS, Rao J, et al. Nanomechanical analysis of cells from cancer patients. Nat Nanotechnolo. 2007;2(12):780-783

11. Faria EC, Ma N, Gazi E, et al. Measurement of elastic properties of prostate cancer cells using AFM. Analyst. 2008;133(11):1498-1500.

12. Gavara N, Chadwick RS. Relationship between cell stiffness and stress fiber amount, assessed by simultaneous atomic force microscopy and live-cell fluorescence imaging. Biomech model mechanobiol. 2016;15(3):511-523.

13. Stolz M, Gottardi R, Raiteri R, et al. Early detection of aging cartilage and osteoarthritis in mice and patient samples using atomic force microscopy. Nat Nanotechnol. 2009;4(3):186-192.

14. Guo Q, Xia Y, Sandig M, et al. Characterization of cell elasticity correlated with cell morphology by atomic force microscope. J Biomech. 2012;45(2):304-309.

15. Calzado Martín A, Mario Encinar, Javier Tamayo, et al. Effect of actin organization on the stiffness of living breast cancer cells revealed by peak-force modulation atomic force microscopy. ACS Nano. 2016;10(3):3365-3374. 